\title{
Unusual Penetrating Injury to the Male Genitalia
}

\author{
S.V. Krishna Reddy \\ Department of Urology, Narayana Medical College, Nellore, India
}

\begin{abstract}
Various self-inflicted foreign bodies have been removed from the lower urinary tract and external male genitalia. In many cases foreign bodies were inserted or applied for autoerotic reasons which are frequently associated with mental health disorders. Here the author reports an unusual case of self insertion of a $6-\mathrm{cm}$ long metal needle at penoscrotal junction of the erect penis for sexual pleasure. The stitching needle was inserted by a 20 -year old male who was too ashamed to admit the motive at first. The caudal end of the needle was palpable at the root of the penis as it was below the urogenital diaphragm. The needle was successfully removed under fluoroscopic guidance with minor surgical exploration and with no complications.
\end{abstract}

Keywords: Penis; Wound and Injuries; Needles; Genitalia

Injuries to male genitalia are mostly iatrogenic in origin or are due to traffic accidents and are sometimes the result of burns, animal bites, ritual circumcision, gun shots, or self-mutilation [1]. In most penetrating injuries of the male genitalia with foreign bodies, the foreign bodies were self-inserted through the urethra owing to psychiatric illness, sexual curiosity, or sexual practice when intoxicated [2]. Insertion of foreign bodies through the urethra is more common than insertion through other areas of the external genitalia [3]. A complete insertion of a $6-\mathrm{cm}$ long needle through the external genitalia at the penoscrotal junction of the erect penis for sexual curiosity is rare. Here the author reports an unusual incident of self-insertion of a metal needle at the penoscrotal junction and its removal without complications.

\section{CASE REPORT}

A 20-year-old-male presented to the emergency department with a history of penetrating injury to the penis by a needle on the same day. The needle was reported to have accidentally pierced the external genitalia at the penoscrotal junction when the penis was erect. There was no history of hematuria. Upon physical examination, the patient was anxious and his vital signs were normal. The caudal end of the needle was just palpable at the penoscrotal junction on local examination.

The results of urinalysis and complete blood examination reports were normal. An X-ray of the pelvis (Fig. 1) showed the needle within the pelvis. Ultrasound examination of the abdomen including the genitalia showed a $6-\mathrm{cm}$ needle piercing the prostate from the root of the penis up to the bladder neck. Because the needle was palpable at the penoscrotal junction and the caudal end of the needle was below the urogenital diaphragm (Fig. 2), an urgent surgical exploration with incision over the palpable end of the needle under fluoroscopic guidance was done. This exploration showed the tip of the caudal end of the needle to be inside the corpus cavernosum at the root of the penis (Fig. 3). The needle was manipulated by holding the caudal end at the penoscrotal junction, which showed that the tip of the needle was in and around the bladder neck by piercing the prostate gland. Endoscopic manipulation was not done in this case. The needle was removed intact through exploration of the bulbous cavernosa (Fig. 4). A pressure dressing was applied to the penis and the patients post operative voiding was normal. When the patient was queried about the possibility of self-in-
Corresponding author: S.V. Krishna Reddy

Department of Urology, Vijaya Hospital, Narayana Medical College, Nellore 524001, Andhra Pradesh, India

Tel: +91-8612321828 / Fax: +91-8612300068 / E-mail: vijaya.nel@gmail.com Submitted: February 4, 2013 / Accepted after revision: March 23, 2013
This is an Open Access article distributed under the terms of the Creative Commons Attribution Non-Commercial License (http://creativecommons.org/licenses/by-nc/3.0/) which permits unrestricted non-commercial use, distribution, and reproduction in any medium, provided the original work is properly cited. 


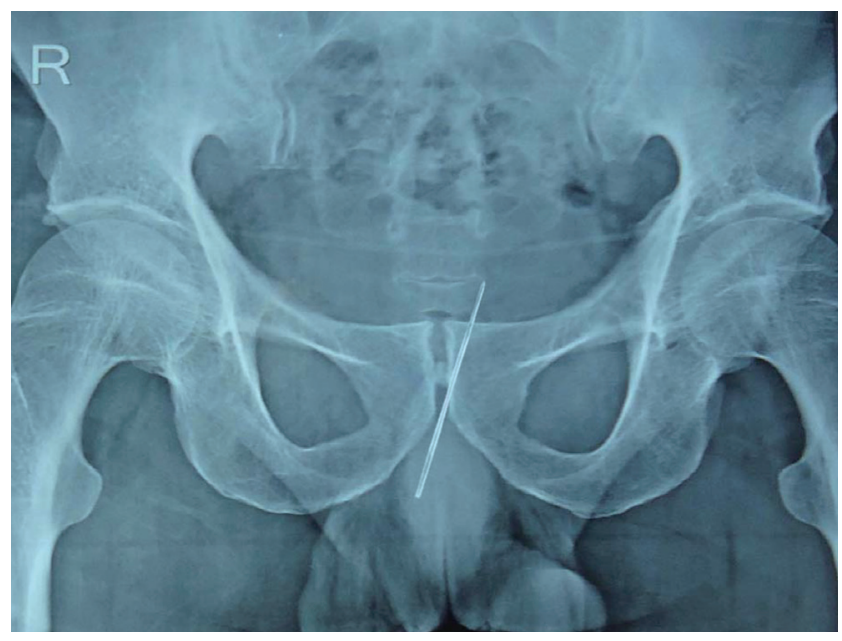

Fig. 1. X-ray of the pelvis showing the needle within the pelvis.

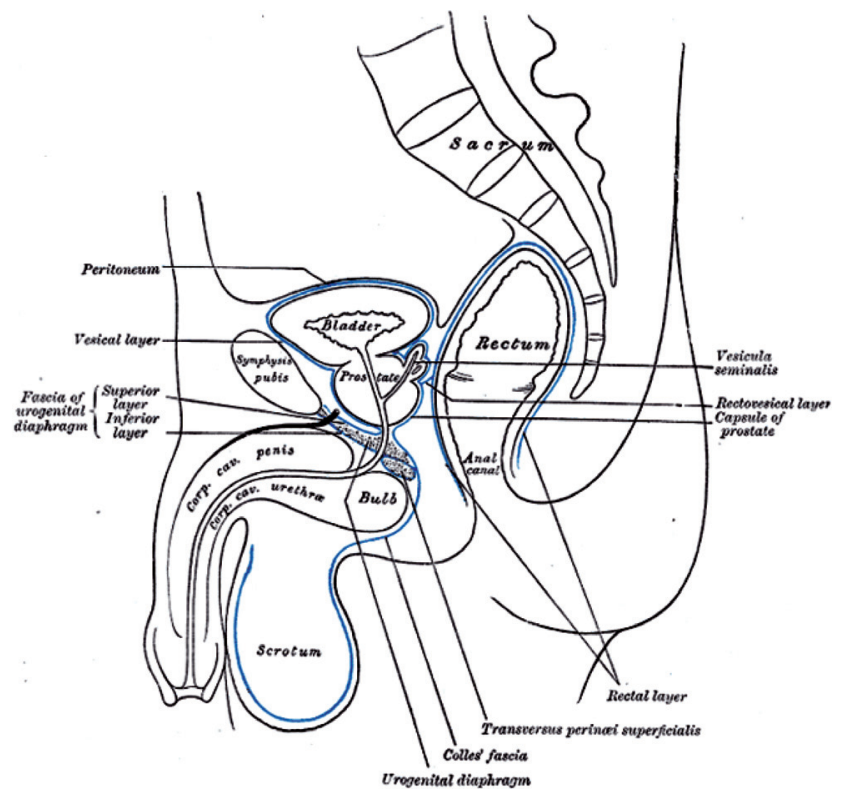

Fig. 2. Diagram showing the urogenital diaphragm.

sertion because accidental injury would be unlikely to push the needle completely through the external genitalia, the patient was too ashamed to admit the cause and was discharged after 2 days. Later, the patient admitted the motive behind the insertion of the needle as sexual gratification. The surgical wound healed well and mild penile edema was noted in the immediate postoperative period. The edema was reduced by a pressure dressing and no complications such as erectile dysfunction or plaque formation occurred during the 1-year follow up after needle removal.

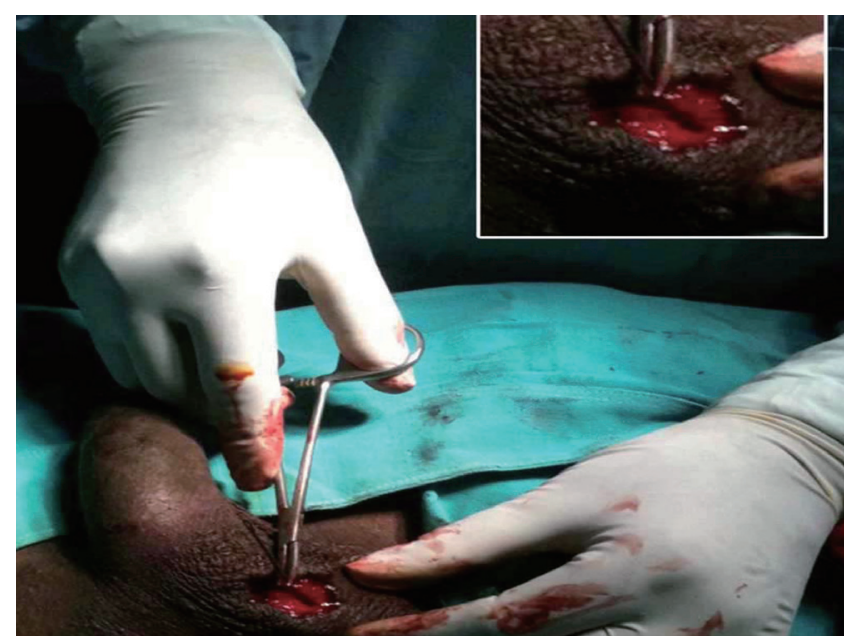

Fig. 3. The caudal tip of the needle inside the corpus cavernosum at the root of the penis.

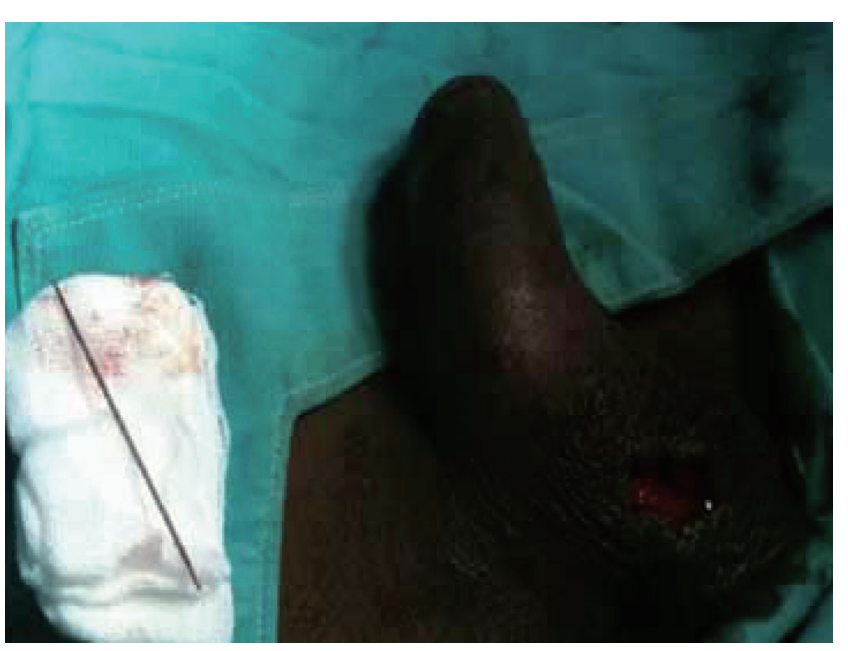

Fig. 4. The removed needle through exploration of the bulbous cavernosa.

\section{DISCUSSION}

The penis is mobile and is protected by its position, but in the erectile state, it is more prone to injury [4,5]. The presence of a foreign body within the male genitalia is rarely due to an accident and is usually the result of self-insertion for erotic purpose [6]. Most cases of a foreign body in male genitalia can be diagnosed through radiologic studies, and computed tomography or ultrasound examination is usually not necessary [7]. A wide variety of unusual foreign bodies, such as a screw, a wire, and a safety pin have been reported in the urethra and urinary bladder [8]. The most suitable method for removing the foreign body depends on the size and depth of penetration of the object in- 
serted into the genitourinary tract. Whenever possible, minimally invasive techniques for removal should be used. Endoscopic manipulation may be necessary when urethral injury is suspected. In our case, the needle was inserted completely for sexual gratification. Because the caudal end of the needle was palpable and was lying below the urogenital diaphragm, endoscopic manipulation was not necessary in this case. Fluoroscopic guidance was useful, and with limited surgical exploration of the corpus cavernosa, the entire needle was removed intact without complications such as erectile dysfunction or plague formation in the follow-up period. To prevent possible future acts with a similar motive, proper counseling and psychiatric referral is required for such adolescent males who are self-inserters so that an appropriate management plan can be formulated [9].

\section{CONFLICT OF INTEREST}

No potential conflict of interest relevant to this article was reported.

\section{REFERENCES}

1. Perovic SV, Djinovic RP, Bumbasirevic MZ, Santucci RA, Djordjevic
ML, Kourbatov D. Severe penile injuries: a problem of severity and reconstruction. BJU Int 2009;104:676-87.

2. Moon SJ, Kim DH, Chung JH, Jo JK, Son YW, Choi HY. Unusual foreign bodies in the urinary bladder and urethra due to autoerotism. Int Neurourol J 2010;14:186-9.

3. Aliabadi H, Cass AS, Gleich P, Johnson CF. Self-inflicted foreign bodies involving lower urinary tract and male genitals. Urology 1985;26:12-6.

4. Amukele SA, Lee GW, Stock JA, Hanna MK. 20-year experience with iatrogenic penile injury. J Urol 2003;170(4 Pt 2):1691-4.

5. Morey AF, Metro MJ, Carney KJ, Miller KS, McAninch JW. Consensus on genitourinary trauma: external genitalia. BJU Int 2004; 94:507-15.

6. Pastor Navarro H, Donate Moreno MJ, Carrion Lopez P, Segura Martin P, Lorenzo Romero J, Pastor Guzman JM, et al. Penile foreign bodies. Arch Esp Urol 2009;62:501-7.

7. Rahman NU, Elliott SP, McAninch JW. Self-inflicted male urethral foreign body insertion: endoscopic management and complications. BJU Int 2004;94:1051-3.

8. Cho DS, Kim SJ, Choi JB. Foreign bodies in urethra and bladder by implements used during sex behavior. Korean J Urol 2003;44:11314.

9. Kenney RD. Adolescent males who insert genitourinary foreign bodies: is psychiatric referral required? Urology 1988;32:127-9. 